\title{
Parecoxib Sodium
}

National Cancer Institute

\section{Source}

National Cancer Institute. Parecoxib Sodium. NCI Thesaurus. Code C73831.

A water-soluble, injectable sodium salt form of parecoxib, an amide prodrug of the cyclooxyg enase II (COX-2) selective, non-steroidal anti-inflammatory drug (NSAID) valdecoxib, with anti-inflammatory, analgesic, and antipyretic activities. Upon intravenous or intramuscular administration, parecoxib is hydrolyzed by hepatic carboxyesterases to its active form, valdecoxib. Valdecoxib selectively binds to and inhibits COX-2. This prevents the conversion of arachidonic acid into prostaglandins, which are involved in the regulation of pain, inflammation, and fever. This NSAID does not inhibit COX-1 at therapeutic concentrations and, therefore, does not interfere with blood coagulation. 